\title{
An innovative method of surgical excision of duodenal diverticulum in a 17-year-old girl
}

\author{
Marta Korycka, Piotr Nogal, Patrycja Sosnowska, Jakub Noskiewicz, Przemysław Mańkowski \\ Department of Paediatric Surgery, Traumatology, and Urology, Poznan University of Medical Sciences, Poznan, Poland
}

ABSTRACT

The first well-documented case of the duodenal diverticulum (DD) was presented by Morgagni in 1762. In 1913 Case presented four cases of radiologically diagnosed DD. The first surgical resection of the duodenal diverticulum took place in 1915 and was performed by Key. The aetiology of the duodenal diverticula is not clear and remains controversial. The existence of the locus minoris resistentiae in the duodenal wall may be the place of mucosal or submucosal tissue layer herniation through the muscular defect. Duodenum is the second most common location of diverticula in the gastrointestinal tract. They appear in about $23 \%$ of the population and are rarely found in children. Surgical treatment is applied only if complications occur. We present a case of a 17-year-old female treated surgically with the use of a minimally invasive laparoscopic method combined with endoscopic control of the surgical field.

KEY WORDS:

surgery, child, paediatrics, gastroenterology, diverticulectomy.

\section{INTRODUCTION}

Duodenal diverticula (DD) are the out-pouchings of the duodenal wall and can be found at any point of it; nevertheless, $90 \%$ of the diverticula occur in the second part of the duodenum. DD represent the second most common diverticula affecting the alimentary tract $[1,2]$. They appear in up to $23 \%$ of the population; however, recent studies have shown that approximately $90 \%$ of them are asymptomatic.

DD are mostly acquired. They rarely occur in infants, children, or adolescents. The peak incidence is between 50-60 years of age, and the risk of the diverticulum presence increases with age. No significant difference in sex distribution of these lesions has been noticed.

Incidentally detected DD do not require any surgical intervention provided they are asymptomatic. A surgical approach is associated with numerous complications and has a mortality rate of about $30 \%$, whereas untreated symptomatic DD can cause a mortality rate of up to $90 \%$ [3].

\section{CASE REPORT}

A 14-year-old female presented with a history of severe recurrent abdominal pain, deteriorating especially under stress. Gastroduodenoscopy showed the presence of lesions in the duodenum, and hence further diagnostics was necessary. A barium study revealed retention of chyme and contrast in the stomach as well as the extraluminal diverticulum in the descending part of the duodenum. Ultrasonography of the abdomen, abdominal X-ray, and colonoscopy showed no abnormalities. The patient was discharged from the hospital, and a check-up was planned in two years' time. Surgical treatment was not implemented due to the decision of the family. In 2014 gastroduodenoscopy revealed slight enlargement of the DD. The results

\section{ADDRESS FOR CORRESPONDENCE:}

Przemysław Mańkowski, Department of Paediatric Surgery, Traumatology, and Urology, Poznan University of Medical Sciences, 27/33 Szpitalna St., 60-572 Poznan, Poland, e-mail: mankowskip@wp.pl 

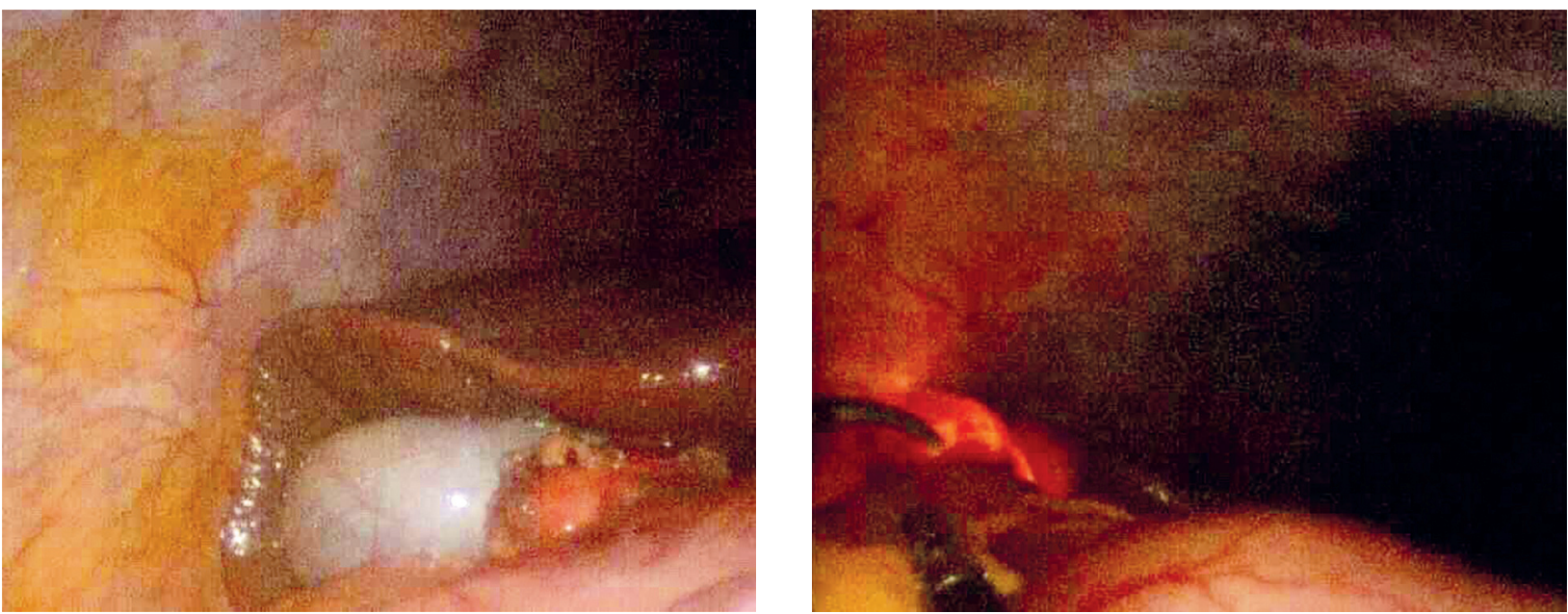

FIGURE 1. Intraoperative view of the transillumination through the diverticulum

of a barium study of the upper gastrointestinal tract (GIT) were comparable to the examination from 2012.

In 2015 at the age of 17 years the patient was finally qualified to surgical excision of the diverticulum. Three trocars were inserted under control of sight. After examination of the abdominal cavity, the diverticulum was dissected and adhesiotomy was conducted. During intraoperative gastroduodenoscopy, the end of the scope was inserted into the diverticulum and transillumination was performed (Fig. 1).

Afterwards, the diverticulum was closed with a use of a linear stapler and evacuated through the troacar. Repeated gastroduodenoscopy showed no sign of duodenal obstruction or perforation. The recovery of the patient was uneventful. She was discharged from the hospital five days after the surgery in a good condition.

\section{DISCUSSION}

The aetiology of true DD is not clear and remains controversial. However, the existence of locus minoris resistentiae in the duodenal wall may be the place of mucosal or submucosal tissue layer herniation through the muscular defect [1]. DD can be divided into two groups: extraluminal (EDD) and intraluminal, whereas EDD are markedly more common. About $75 \%$ of the lesions are located periampullary [1]. In this case localisation was typical and the diverticulum was classified as EDD. Once formed the diverticulum can become larger in time [1]. In our patient's case there was a slight enlargement noticed in gastroduodenoscopy.

DDs are usually located in the retroperitoneal space; therefore, both the onset of symptoms and the diagnosis are often delayed. In only about $10 \%$ of cases DDs are symptomatic. When they occur, symptoms include abdominal pain, bezoar formation, biliary or pancreatic duct obstruction, haemorrhage, duodenal obstruction, and perforation. Hence, this condition enters in the differential diagnosis of acute abdomen. In some cases it was described that the obstruction of the diverticulum was associated with prior ingestion of a foreign body. It was also noted that some DD caused secondary acute pancreatitis with the typical clinical presentation of the disease [4-6]. Complications of duodenal diverticula are rare, but they can be severe [2-5]. Around 5\% of patients with DD have to be treated surgically due to the complications presented above. However, in this case the surgery was performed in order to avoid possible complications.

DD are often found accidentally during GIT examination or surgeries performed for other reasons [4, 7-9]. According to recent studies, the following diagnostic methods proved useful during the perioperative period: UGI series; CT scan, and endoscopy. Upper GI studies and endoscopy can be of some assistance, but mainly in stable patients. Otherwise, the diagnosis is usually made with intraoperative exploration [6]. Our patient had imaging and endoscopic studies performed due to suspicion of early signs of celiac disease. Earlier she presented symptoms of chronic abdominal pain and diarrhoea.

Indications to the surgery described in the literature comprise: chronic abdominal pain with inflammation, bleeding, perforation, obstructive jaundice, and gastric outlet obstruction $[2,4-6,9]$. In our patient's case the indication for the procedure was aggravating abdominal pain and progression of the lesion in size.

There are several types of treatments described, both surgical or conservative $[2,4-7,10]$. The procedure of choice is duodenotomy with the excision of the diverticulum. The method of treatment should be chosen according to the patient's condition and the localisation of the DD: segmental duodenectomies, pylorus-preserving pancreaticoduodenectomy ( $\mathrm{p}-\mathrm{p}$ Whipple), and diverticulectomies have been described $[2,6,10]$. In the presented case the surgery was performed with the innovative use of a laparoscopic method combined with endoscopic control of the surgical field. Intraoperative gastroduodenoscopy was necessary to identify the DD and to exclude injuries affecting anatomical structures, and also to ascertain that the 
duodenal lumen will not be iatrogenically constricted. Additionally, the combined use of gastroduodenoscopy and transillumination through the wall of the DD allowed us to preserve healthy tissue margin and significantly improved the mobility of the dissected structures. Furthermore, intraoperative transillumination enabled selection of the most optimal plane of dissection with the use of a linear stapler. We did not find a similar approach in the literature.

There are a few cases in the literature of DD treated with the use of antibiotics and percutaneous drainage after perforation of the diverticulum $[2,10]$. In some non-complicated cases a conservative approach was introduced $[2,10]$. Based on our experience, this kind of solution may alleviate the symptoms and decrease their recurrence. However, it should be only temporary, in expectation of surgical treatment, which remains the only guarantee of full recovery.

The surgery was performed in order to prevent longterm complications of DD. Nonetheless, enlargement of the diverticulum and recurrent abdominal symptoms relevantly contributed to the decision of surgical intervention.

\section{DISCLOSURE}

The authors declare no conflict of interest.

\section{REFERENCES}

1. Afridi SA, Fichtenbaum CJ, Taubin H. Review of duodenal diverticula. Am J Gastroenterol 1991; 86: 935-938.

2. Barillaro I, Grassi V, De Sol A, et al. Endoscopic rendez-vous after damage control surgery in treatment of retroperitoneal abscess from perforated duodenal diverticulum: a technical note and literature review. World J Emerg Surg 2013; 8: 26.

3. Schnueriger B, Vorburger SA, Banz VM, et al. Diagnosis and management of the symptomatic duodenal diverticulum: a case series and a short review of the literature. J Gastrointest Surg 2008; 12 1571-1576

4. Saavedra VL, Kane TD, Garrity SH. Surgical Management of Symptomatic Intraluminal Duodenal Diverticulum in an Adolescent. Glob Pediatr Health 2018; 5: 2333794X18755240.

5. Khan K, Saeed S, Maria H, et al. Duodenal Diverticular Perforation after Small Bowel Obstruction: A Case Report. Case Rep Surg 2018; 2018: 6197828 .

6. Yeh T-C. Laparoscopic resection of perforated duodenal diverticulum - A case report and literature review. Int J Surg Case Rep 2016; 28: 204-210.

7. Gupta P, Debi U, Sinha SK, Prasad KK. Upper gastrointestinal barium evaluation of duodenal pathology: A pictorial review. World J Radiol 2014; 6: 613-618.

8. Kouraklis G, Glinavou A, Mantas D, et al. Clinical implications of small bowel diverticula. Isr Med Assoc J 2002; 4: 431-433.

9. Coelho JC, Sousa GS, Salvalaggio PR. Laparoscopic treatment of duodenal diverticulum. Surg Laparosc Endosc 1999; 9: 74-77.

10. Martinez-Cecilia D, Arjona-Sanchez A, Gomez-Alvarez M, et al. Conservative management of perforated duodenal diverticulum: a case report and review of the literature. World J Gastroenterol 2008; 14: 1949-1951. 